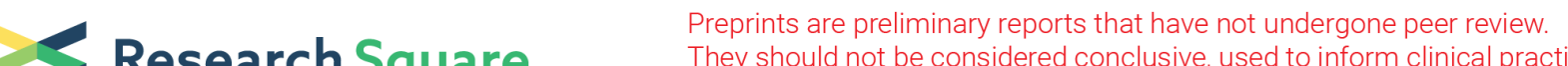 $\begin{array}{ll}\text { Research Square } & \text { They should not be considered conclusive, used to inform clinical practice, } \\ \text { or referenced by the media as validated information. }\end{array}$
}

\section{ACE2 Protein-Protein Interaction Networks Reveal Potential Druggable Targets For SARS-CoV-2}

\section{Qiaoxi Xia}

Shantou Central Hospital

Xiao Zhou

Shantou Central Hospital

\section{Mantong Chen}

Shantou University Medical College

Ling Lin

Shantou University Medical College

Yan Zhao

Shantou Central Hospital

Xiaoqi Zheng

Shantou University Medical College

Zhongte Peng

Shantou University Medical College

Meihui Huang

Shantou Central Hospital

Shaohong Wang

Shantou Central Hospital

Zepeng Du ( $\square$ zepdu@126.com )

Shantou Central Hospital https://orcid.org/0000-0002-9595-2439

\section{Research}

Keywords: ACE2, protein-protein interaction network, drug-protein network, druggable target

Posted Date: September 22nd, 2021

DOI: https://doi.org/10.21203/rs.3.rs-54096/v2

License: (9) This work is licensed under a Creative Commons Attribution 4.0 International License. Read Full License 


\section{Abstract}

Background: The novel coronavirus SARS-CoV-2 pandemic has infected more than 130 million people, killed over 2.3 million so far. Currently, no effective drugs are available to treat this infectious disease, due to limited knowledge of the molecular mechanisms of SARS-CoV-2 infection. ACE2 (angiotensin I converting enzyme 2) has long been identified as the major receptor for coronavirus entry the host cells.

Methods: In this study, we constructed the protein-protein interaction networks (PPIN) based on ACE2 and its interacting proteins, considering with the expression alternation and co-expression relationship. The potential drugs targeting the proteins in the PPIN were explored.

Results: ACE2 and its interacting proteins AAMP and HRAS are obviously increased, and their PPIN show distinguishing expression patterns during the COVID-19 progression. At least six pathways are activated for the host cell in the response to the virus. Moreover, drug-target networks were built to provide important clues to block ACE2 and its interacting proteins. Except the reported four drugs for ACE2, its interacting protein CALM1 and HRAS are great potentially druggable. We also considered the path initiated from ACE2 to nucleus by cascades of interaction, especially for the transcription factors in the PPIN which are also druggable.

Conclusion: In summary, this study provides new insight into the disruption of the biological response to virus mediated by ACE2, but also its cascade interacting proteins when considering of PPIN.

\section{Introduction}

The outbreak of global SARS-CoV-2 pandemic has profoundly threatened the health of billions of individuals, overburdened local health care systems and millions of lives have lost by the end of May 2021 (https://coronavirus.jhu.edu/map.html). The most distinguishing clinic character of COVID-19 patients is a dysfunction of breath primarily due to acute respiratory distress syndrome, causing a greater risk of mortality [1]. The first step of viral infections is the entry of the virus of the host cell, following the replication multiple viral copies using the host cellular machinery. It has been widely acknowledged that angiotensin-converting enzyme-2 (ACE2, EC 3.4.17.23) acts as a major receptor for COVID-19 to gain intracellular entry into the host cells [2]. ACE2 is a membrane-bound protein, with a $\mathrm{NH}_{2}$-terminal domain comprises the catalytic site oriented extracellularly [3]. ACE2 is normally expressed in many human tissues, including lung, small intestine, heart, brain stem, nasal and oral mucosa [4].

The mechanism for COVID-19 causes lung dysfunction has not been fully proposed. ACE2 is considered as a major receptor for coronavirus entry into the cells, which causes diffuse alveolar damage through imbalances in the renin-angiotensin system. These immunologic reactions in severe COVID-19 may characterize the cytokine storm phenomenon: a massive release of cytokines, such as interleukin (IL)-6, IL-1, IL-8 and TNFa, from host cells and immune cells, such as monocytes and dendritic cells $[5,6]$. These inflammatory cells and factors will severe respiratory complications, like ARDS (Acute respiratory distress 
syndrome), characterizing acute diffuse alveolar damage, pulmonary oedema and formation of hyaline membranes [7].

However, the detailed of molecular mechanism of how ACE2 is stimulated by virus and the progress of phenotype in host cell remain unclear. Many proteins carry out multiple biological functions through the interaction with other proteins, which maybe predicted by the interacting proteins. Analyses based on protein-protein interaction networks (PPINs) have become a prevalent method for the high-throughput data, especially the protein interaction data [8]. PPINs analysis can systematically integrate single or multiple high-throughput data to obtain a global understanding of cellular functions or processes under various conditions, e.g., different tumor sub-types, cancer progressive stages and cell sub-groups $[9,10]$.

In this study, the PPINs for ACE2 and its interacting proteins was constructed, and the expressed change and expression pattern during the COVID-19 progression were integrated into the networks. Moreover, a drug-target network was built to provide the clues to block the potential molecular path initiated from ACE2.

\section{Methods And Materials}

\section{Search of proteins that interacts with ACE2}

The reported interacting proteins of ACE2, confirmed by previous publications, were obtained from two databases of BioGRID (https://thebiogrid.org/) and MINT (https://mint.bio.uniroma2.it/). These proteins were curated to obtain a unique interacting protein list.

\section{Construction of the protein-protein interaction network (PPIN)}

The latest known human protein-protein interaction data were obtained from several databases, including BioGRID (http://thebiogrid.org/), HPRD (http://www.hprd.org/), MINT (https://mint.bio.uniroma2.it/), and IntAct (http://www.ebi.ac.uk/intact/). These protein interaction data were integrated and obtained a unique clean dataset after the remove of redundancy, which contained the up today human proteinprotein interactions. A total of 24,046 unique proteins and 438,656 interactions were contained in this clean dataset, which was applied as the parental PPIN from that new or sub-PPINs were constructed by Cytoscape [11]. Firstly, a sub-network was built by mapping ACE2 and its interacting proteins were mapped to the parental PPIN as the seed proteins, to extract of their first class directly interacting proteins. This subnetwork is named "ACE2 Full-PPIN". Secondly, a smaller PPI sub-network was illustrated, which was named "ACE2 Core-PPIN", in which ACE2 and its interacting proteins are linked through one or more partner proteins.

\section{Topological parameters analyse for the PPIN}

NetworkAnalyzer was applied to analyze the topological parameters, which would provide insight into the complex network in terms of its organization and structure [12]. As one of most important property of many large networks, the power law of distribution of node degrees was analyzed as we described 
previously [13]. The node degree of a node $n$ is defined as the number of nodes connected with $n$. When considering the degree of all nodes in the network, we will have the equation of $p(k)=N k / N$, with $N k$ $=$ number of nodes with degree $\mathrm{k}$. Moreover, several other important topological parameters, such as closeness centrality, shortest path length and topological coefficients, were analyzed for visualization.

\section{Subcellular distribution of the PPIN}

Subcellular locations for the proteins in the "ACE2 Full-PPIN" were downloaded from the HPRD database and served as a node attribute in the network as we previously described [14]. If some proteins are annotated by multiple locations, e.g., membrane and nucleus, were merged as membrane/nucleus. The nodes in the "ACE2 Full-PPIN" were re-distributed into different layers by Cerebral plugin according to the annotated subcellular localizations, generating a pathway-like graph remaining their physically interactions [15].

\section{Enrichment analyses for functions and pathways of network}

Functional enrichment analysis of the "ACE2 Full-PPIN" was performed in DAVID database (https://david.ncifcrf.gov/) to identify the enriched Gene Ontology (GO) "Biological Process" term. GO terms with a statistically significant $P$-value less than 0.05 were remained for visualization using ggplot2 R package.

\section{Expression pattern for the Core-network in COVID infected lung cells}

GSE147507, an expression dataset containing SARS-CoV-2 infected normal lung cells, were obtained from GEO database (https://www.ncbi.nlm.nih.gov/geo), which was submitted by Blanco-Melo et al. [16]. In this dataset, primary human lung epithelium (NHBE) was mock treated or infected with SARS-CoV-2 by biological triplicates. To mimic the effect of inflammatory factor, NHBE cell was treated with human interferon-beta (INFB) with the timepoint was set as $4 \mathrm{~h}, 6 \mathrm{~h}$ and $12 \mathrm{~h}$. The RNA-seq data was normalized and the differentially expression were analyzed by SangerBox (http://soft.sangerbox.com/). The Pearson expression correlation for each pair proteins in the "ACE2 Core-PPIN" were analyzed by an R script, according to the expression data. The expression fold changes and correlations were integrated into the "ACE2 Core-PPIN" as the node attribute, to illustrate the dynamic changes of the Core-PPIN under different conditions. The correlation matrixs and their expression profile were clustered by Cluster 3.0 and illustrated by TreeView [17].

\section{Activated pathways analyses}

The expression matrix for the proteins in the "ACE2 Core-PPIN" was submitted to the Pathview (https://pathview.uncc.edu/) for visualization, which provides easy interactive access, and generates high quality pathway graphs [18].

\section{Drug-target network construction}


The latest release of DrugBank (version 5.1.6, https://www.drugbank.ca/) was downloaded, which contains 13,580 drug entries, including FDA approved small molecule drugs, biologics, 131 nutraceuticals, 6,376 experimental drugs, as well as 5,223 non-redundant target proteins. An intersection was obtained between the proteins in "ACE2 Full-PPIN" and target proteins from DrugBank, to construct a drug-target network.

\section{Shortest path from ACE2 to transcription factor}

The shortest path problem is any possible but the minimized path(s) from one node to another given node in the network. The proteins in the "ACE2 Full-PPIN" was compared the human transcription factors (http://humantfs.ccbr.utoronto.ca/) to identify the transcription factors in this network. The path from ACE2 to these transcription factors were analyzed by R "igraph" package as we previously described [19]. The visualization for the path from ACE2 to transcription factors, as well as their targeted drug were performed by Cytoscape.

\section{Results}

\section{Visualization of the PPIN for ACE2 and its interacting proteins}

The currently known interacting proteins of ACE2 were obtained from the BioGRID and other databases. At the beginning of this study, there are 12 reported ACE2 interacting proteins, with information shown in Table 1 (Fig. 1A). A PPIN was built, using ACE2 and its interacting proteins as the seed nodes to extract all their interacting proteins from the parental PPIN, which was called as the "ACE2 Full-PPIN" (Fig. 1B). The "ACE2 Full-PPIN" contained 1,318 nodes and 1,292 edges, suggesting that ACE2 and its interacting proteins can connect with thousands partner proteins by cascaded interactions to exceed the biological effects of solo protein. Three top genes with the highest number of edges were HRAS (620 edges), CALM1 (472 edges) and CAT (119 edges).

In the "ACE2 Full-PPIN", we found multiple ACE2-interacting protein were connected by many common partner proteins. To better illustrate their inner links, another smaller PPI sub-network, named as the "ACE2 Core-PPIN" was constructed by showing ACE2 and its interacting proteins linked by one or more partner proteins, in which the nodes with only one interaction in the "ACE2 Core-PPIN" were removed (Fig. 1C). This "ACE2 Core-PPIN" contained 80 nodes and 154 edges, in which ACE2 and its interacting proteins were illustrated in light pink, while the partner proteins were indicated in light blue. Interestingly, it shown HRAS and CALM1 have the highest number of shared nodes. This indicates that HRAS and CALM1 would have the greatest potential to transduce the stimulus from ACE2 (Fig. 1C). Moreover, we consider these common nodes between ACE2 and its interacting proteins might act as switch proteins, then determine the direction of molecular signal paths by their expression level, and co-expression correlation strength as well.

\section{Topological structure of the "ACE2 Full-PPIN"}


A real biological network, including PPIN, is distinguishable from any chaos or random networks by its distinct structural properties. Many real, complex networks are characterized as "scale-free", with a power law degree distribution [20]. For "ACE2 Full-PPIN", the distributions of node degree followed an

approximate power law, with the equation $y=288.18 x^{-1.07}$ and an $R^{2}=0.833$ (Additional file $1 \mathrm{~A}$ ). The shortest path length (the minimum number of links from one protein to another protein) of "ACE2 FullPPIN" was shown as mainly arranged from 2 and 3 step lengths (Additional file 1B). It also suggests that one protein can contact another protein by only a few nodes, enabling the transformation of different protein complexes or/and component maintenance. Topological coefficient is a measure for the extent to which a node shares neighbors with other nodes. A topological coefficient of 0 is assigned to nodes which have one or no neighbors (Additional file 1C). Closeness centrality measures how fast the flow of information would be through a given node to other nodes in the network, considering the efficiency of signal transduction in the network. The centrality curve is flat when the number of links less than 10 , but it gradually increases with more nodes have the links >10 (Additional file 1D).

\section{Signal transduction from cell surface to nucleus through the PPIN}

Evidence suggest that a protein might perform diverse functions depending on its cellular translocation, except the interactions with other proteins [21,22]. So, the subcellular location and/or translocation become critical when considering its function, for the PPIN as well. After re-distribution, the "ACE2 FullPPIN" was divided into 11 layers and the percentage from extracellular to nucleus as follows: secreted (2.43\%), membrane $(22.53 \%)$, cytoskeleton $(0.15 \%)$, cytoskeleton/cytoplasm $(0.23 \%)$, cytoplasm (19.35\%), secreted/nucleus ( $0.46 \%)$, membrane/nucleus $(0.23 \%)$, cytoskeleton/nucleus $(0.61 \%)$, cytoplasm/nucleus (18.06\%), nucleus (8.19\%) and uncertain $(27.77 \%$, distribute near the interacting proteins) (Fig. 2A). It shown that near $20 \%$ nodes in this PPIN have multiple subcellular locations. It also strongly suggests that the ACE2 might transduct stimulus from the extracellular/membrane into the cytoplasm, till to the nucleus, making up various non-classical pathways through cascades of interactions.

\section{Functional enrichment of the "ACE2 Full-PPIN"}

As a tightly connected protein network, we presumed that ACE2 and its interacting proteins are involved in various biological processes, especially in the pathology of COVID-19, through cascaded interactions to amplify their biological effects. To test this speculation, GO "Biological Process" enrichment analysis of "ACE2 Full-PPIN" was performed, acquiring more than a hundred of significantly enriched GO terms (data not shown). Two big groups GO terms caused us a great interest (Fig. 2B). One group relates to virus process, including "GO:0046718 viral entry into host cell", "GO:0039694 viral RNA genome replication", "GO:0019083 viral transcription" and "G0:0016032 viral process". The other interested group is about immunity, includes "G0:0050900 leukocyte migration", "G0:0031295 T cell costimulation", "GO:0050690 regulation of defense response to virus by virus" and "GO:0042110 T cell activation". These detailed significant GO terms with their enriched genes are listed in Additional file 2. 


\section{Dynamic expression pattern for the Core-PPIN in COVID infected lung cells}

In living cells, PPINs are not static, but dynamically change under different stimulus, or different stages of disease. For this aim, the expression data of GSE147507 containing different conditions of lung cells was obtained. Two important parameters for "ACE2 Core-PPIN" were analyzed, the expression log2(foldchange) (logFC), and the co-expression correlation coefficient of each protein interaction. Then, the logFC and correlation coefficient were imported into the network as a node attribute and an edge attribute, respectively, to observe the dynamic changes in various treatments. In the Series 1 of GSE147507, the primary human lung epithelium (NHBE) was infected with SARS-CoV-2 (USA-WA1/2020) with mock treated as a control (Fig. 3A). ACE2 is significantly increased, followed by its interacting protein AAMP and HRAS. On the other hand, MYC in the network is also significantly upregulated (Fig. 3B). For expression coefficient, there are near $70-80 \%$ of positive co-expression in mock NHBE, while it increases to more than $90 \%$ positive in SARS-CoV- 2 treated cells. The co-expression patters between normal and treated are rather different, as indicated by the heatmap (Fig. 3C-D). In the Series 9 of GSE147507, NHBE were treated with human interferon-beta at the time point of $4 \mathrm{~h}, 6 \mathrm{~h}$ and $12 \mathrm{~h}$ to mimic the stimulus of inflammation. After the treatment, ACE2 and several interacting proteins, such as CALM1, DLEU2, ISYNA1 and NTS are obviously increased in the time series. While GHRL is consistently decrease. HRAS is upregulated gradually from $4 \mathrm{~h}$ to $12 \mathrm{~h}$, though not reach a very high expression level. For the coexpression relationship, it is interesting to point out that both CALM1 and HRAS have positive correlation with their interacting proteins in the control, but only CALM1 remains most positive correlation, while HRAS mostly turns to negative correlation with its interacting proteins (Fig. 4A-D, left panel). For global co-expression pattern as shown by the heatmaps, the total positive correlations are reduced while the total negative correlations are increased (Fig. 4A-D, right panel). On the other hand, the expression pattern of "ACE2 Core-PPIN" could distinguish the control group from the three treatment groups (Additional file $3)$.

\section{Activated pathways in IFN stimulated lung cells}

To exam what kinds of pathway are active during the mimic of COVID-19 stimulus, the expression matrix of NHBE treated with IFN was analyzed by Pathview, which maps omics data to the KEGG pathway. There are six significant enriched pathways, including "PI3K-Akt signaling pathway", "Focal adhesion", "ECMreceptor interaction", "Cell adhesion molecules", "Antigen processing and presentation", and "Regulation of actin cytoskeleton" (Additional file 4). To our great interesting, the genes mapped to the pathways are mostly increased, suggesting these pathways are activated during the mimic of COVID-19 inflammation environment.

\section{The proteins in Full-network are potential drug targets}

Though many scientists and doctors are working hand to find ways to cure the COVID-19, including the drugs targeting ACE2 itself, or the replication of SARS-CoV-2 virus. We consider it would be great helpful to find drugs that target the ACE2 PPIN, to restrict the biological activity stimulated by the virus, thus reduce the virus replication and spread of the SARS-CoV-2. To achieve this, we search the DrugBank 
database and constructed drug-protein target networks. Currently, there are four registered drugs targeting ACE2, there are DB01611 (Hydroxychloroquine), DB05203 (SPP1148), DB15643 (N-(2-Aminoethyl)-1aziridineethanamine), DB00608 (Chloroquine) (though some drugs are now in great controversy) (Figure $6 \mathrm{~A})$. On the other hand, five ACE2 interacting proteins (CALM1, HRAS, AGT, ISYNA1 and CAT) are reported druggable. Among them, CALM1 (calmodulin 1) has the largest number of 29 drugs. The famous signal molecular HRAS (HRas proto-oncogene, GTPase) has five tested drugs (Fig. 5A). By targeting the "ACE2 Full-PPIN", there are 2075 nodes (1728 drugs and 347 proteins) and 2396 edges (targeting relations) in the drug-protein network (Fig. 5B). At least $26 \%$ of proteins in "Full-PPIN" are druggable, suggesting a great potential for treatment. The top 10 proteins have highest number of drugs, and the top 10 drugs have the highest number of target proteins are shown in the list (Fig. 5C). ESR1 (estrogen receptor 1) has the highest of 118 drugs, while DB12010 (Fostamatinib) targets the highest number (61) of proteins. Some drugs have more than one targets. The detailed information about the drugs in these networks (Fig. 5 ) is provided in the Additional file 5.

\section{The shortest paths from ACE2 to the downstream transcription factors}

Usually an external stimulus, or the overexpression/knockdown of one gene, could cause a wide range change in gene expression profile. We presumed the involved transcription factors play vital roles in the alternation of gene expression profile. We applied the shortest path algorithm to illustrate how ACE2 reaches a specific transcription factor by the cascades of interaction in the PPIN. 37 transcription factors are present in the "ACE2 Full-PPIN". Consistent with the shortest path distribution described above, there are only two steps from ACE2 to these transcription factors (Fig. 6A), suggesting a quick response is exist from extracellular stimulus into nucleus, triggering the change of expression profile, then the change of cellular activities. These transcription factors are also ideal targets for treatment. So we construct a small PPIN, in which the drugs target the ACE2-TF. There 278 drugs in the small PPIN, targeting the 37 transcription factors (Fig. 6B). It is critical to understand why a wide range alternation of expression profile is caused when a gene overexpression/knockdown. Shortest path algorithm was applied to identify all possible paths from ACE2 to the transcription factor in the PPIN, which are most effective and economical cellular information transduction.

\section{The verification cohort from public data}

During the preparation of this manuscript, Gordon et al. reported a comprehensive SARS-CoV-2 protein interaction map [23]. They expressed 26 of the 29 SARS-CoV-2 proteins to identify their interacting human proteins. They reported 332 high-confidence SARS-CoV-2-human protein-protein interactions, in which 66 druggable human proteins or host factors are targeted by 69 compounds[23]. To test the reliable of ACE2 based PPIN in this study, we compared the "ACE2 Full-PPIN" with the 332 SARS-CoV-2 interacting proteins, and found 44 intersection proteins which were used to construct a co-network containing 510 nodes and 1264 edges (Additional file 6A). Among these nodes, at least six proteins (CALM1, HRAS, DEFA5, CAT, S and ISYNA1,6/12 ACE2 interacting proteins) from "ACE2 Full-PPIN", and fifteen SARS-CoV2 coding proteins are presented. After reduce the nodes with single connection, a core smaller PPIN was 
obtained to show a clear relationship between ACE2 interacting proteins and SARS-CoV-2 coding proteins (Additional file 6B). It also shows that HRAS and CALM1 are still the nodes with a large number of interactions, suggesting the consistent between the networks in this study and networks from Gordon, D. E. et al.

\section{Discussion}

COVID-19 is still a prevalent global pandemic that has already caused more than 3 million deaths worldwide so far. Biological activities, includes stimulus response and signal transduction, are built on protein physically interactions. In this study, several high confident PPINs were constructed based on the ACE2 and its interacting proteins. Many proteins might link through partner proteins, which could act as switch protein to determine the direction of signal transduction.

Many previous reported biological networks are static networks, which only present a specific state or one time point. It has been widely aware that cellular system, including the PPIN, is highly flexible in the response to environmental stimulus, which enables the cells adapt to different pathological stages or physiological conditions [24]. To measure the co-expression of each pair of interacting proteins in expression profiles, Pearson correlation coefficient is a popular correlation method [25]. In this study, we not only constructed the static PPI network for ACE2 and its interacting proteins, but also the dynamic networks by regarding their expression profiles in different kinds of conditions mimic COVID-19 progress. Several proteins are consistent change during the progress, showing a dynamic co-expression pattern.

Since there is no single effective drug for COVID-19 by now, we consider whether there are multiple drugs available for the ACE2 based PPIN to disturb the biological activities caused by the virus. Inspire by this hypothesis, we found hundreds of drugs targeting ACE2 and its cascaded interacting proteins. With the development of network pharmacology, the drug discovery paradigm has changed from the traditional model "one drug $\rightarrow$ one target $\rightarrow$ one disease"' to the network mode "multi-drugs $\rightarrow$ multi-targets $\rightarrow$ multidiseases" [26, 27]. One successful application of drug cocktails is the clinical treatment of HIV/AIDS patients[28]. The cocktail treatment strategy has been also applied in other diseases, such as breast cancer and leukemia [29-31]. To overcome this combinatorial explosion problem, Zimmer et al. have developed a model that predicted the effects of cocktails at all doses based on measuring pairs of drugs [32]. So we believe that cocktails drug from the ACE2 drug-target network might provide important clues to treat the virus in the clinic trial. Some of ACE2 interacting proteins are reported as drug targets in different diseases. HRAS is a proto-oncogene which overexpresses in many tumors. Tipifarnib is a potent and highly selective inhibitor of farnesyltransferase, a critical enzyme requisite for HRAS activation and now a phase II trial in urothelial carcinoma is carried on [33]. CALM1 is one of kind of the EF-hand calcium-binding protein, which modulates the function of ion channels by calmodulin regulation. CBP501, a drug currently in phase II clinical trial for lung cancer, may sensitize tumors to the chemotherapeutic agents bleomycin and cisplatin by inhibiting CALM1[34]. 
It usually causes a large scale of alternation in gene expression profile when the cells are stimulated. We presumed the transcription factors or transcriptional regulators in the PPIN play crucial roles in this expression profile change. We also speculated that the information transmitted from one protein to another would also employ the most economical ways, through the cascaded of PPI interactions. So, we identified there are only two steps for ACE2 to transcription factors, to cause the alternation of gene expression profile to make the cell adapt to the stimulus. These transcription factors are considered as drug targets. The transcription factor MYC, obviously upregulates both in the COVID-19 and IFN treated lung cells, is a promising therapy target for multiple human cancers. Small molecules are found capable of repression the transcriptional regulation of c-Myc, including IIA6B17, NY2267, and 28RH-NCN-1, which inhibit MYC binds to DNA [35]. Based the above analyses, we propose a working model for ACE2 and its interacting proteins, showing the cascaded interaction and their potential drugs for treatment (Fig. 7).

\section{Conclusion}

In summary, this study provides new insight into the disruption of the biological response to SARS-CoV-2 mediated by ACE2, but also its cascade interacting proteins when considering of PPIN.

\section{Declarations}

\section{Funding}

This work was supported by grants from the National Natural Science Foundation of China (No.81672473, No.81502138), the Science and Technology Program of Guangdong (No.2017A030313181).

\section{Acknowledgement}

We thank Dr. Bingli Wu for the research suggestions and proofreading the manuscript.

\section{Authors' Contributions}

Qiaoxi Xia and Mantong Chen completed the analysis, interpretation, and writing of the manuscript. Xiao Zhou and Ling Lin performed the drawing of graphs in the manuscript. Yan Zhao, Xiaoqi Zheng, Meihui Huang and Shaohong Wang carried out the data collection of the manuscript. Zepeng Du finished the concepts, design and review of the manuscript.

\section{Availability of data and materials}

Public data from NCBI were applied in this article, which might involve human participants, and the Informed content were available from the original papers.

\section{Ethics approval and consent to participate}


Not applicable.

\section{Consent for publication}

Not applicable

\section{Conflicts of interest}

All the authors declare no conflict of interest.

\section{References}

1. Guan WJ, Ni ZY, Hu Y, Liang WH, Ou CQ, He JX, Liu L, Shan H, Lei CL, Hui DSC, et al. Clinical Characteristics of Coronavirus Disease 2019 in China. N Engl J Med. 2020;382(18):1708-20.

2. Li Y, Zhou W, Yang L, You R. Physiological and pathological regulation of ACE2, the SARS-CoV-2 receptor. Pharmacol Res. 2020;157:104833.

3. Chappell MC. Biochemical evaluation of the renin-angiotensin system: the good, bad, and absolute? Am J Physiol Heart Circ Physiol. 2016;310(2):H137-52.

4. Zou X, Chen K, Zou J, Han P, Hao J, Han Z. Single-cell RNA-seq data analysis on the receptor ACE2 expression reveals the potential risk of different human organs vulnerable to 2019-nCoV infection. Front Med. 2020;14(2):185-92.

5. Mahmudpour M, Roozbeh J, Keshavarz M, Farrokhi S, Nabipour I. COVID-19 cytokine storm: The anger of inflammation. Cytokine. 2020;133:155151.

6. Meftahi GH, Jangravi Z, Sahraei H, Bahari Z. The possible pathophysiology mechanism of cytokine storm in elderly adults with COVID-19 infection: the contribution of "inflame-aging". Inflamm Res 2020.

7. Pericas JM, Hernandez-Meneses M, Sheahan TP, Quintana E, Ambrosioni J, Sandoval E, Falces C, Marcos MA, Tuset M, Vilella A, et al. COVID-19: from epidemiology to treatment. Eur Heart J. 2020;41(22):2092-112.

8. Lotfi Shahreza M, Ghadiri N, Mousavi SR, Varshosaz J, Green JR. A review of network-based approaches to drug repositioning. Brief Bioinform. 2018;19(5):878-92.

9. Barabasi AL, Gulbahce N, Loscalzo J. Network medicine: a network-based approach to human disease. Nat Rev Genet. 2011;12(1):56-68.

10. Zhang Q, Li J, Xue H, Kong L, Wang Y. Network-based methods for identifying critical pathways of complex diseases: a survey. Mol Biosyst. 2016;12(4):1082-9.

11. Baryshnikova A. Exploratory Analysis of Biological Networks through Visualization, Clustering, and Functional Annotation in Cytoscape. Cold Spring Harb Protoc 2016, 2016(6).

12. Doncheva NT, Assenov Y, Domingues FS, Albrecht M. Topological analysis and interactive visualization of biological networks and protein structures. Nat Protoc. 2012;7(4):670-85. 
13. Wu B, Li C, Du Z, Yao Q, Wu J, Feng L, Zhang P, Li S, Xu L, Li E. Network based analyses of gene expression profile of LCN2 overexpression in esophageal squamous cell carcinoma. Sci Rep. 2014;4:5403.

14. Du Z, Wu B, Xia Q, Zhao Y, Lin L, Cai Z, Wang S, Li E, Xu L, Li Y, et al. LCN2-interacting proteins and their expression patterns in brain tumors. Brain Res. 2019;1720:146304.

15. Barsky A, Gardy JL, Hancock RE, Munzner T. Cerebral: a Cytoscape plugin for layout of and interaction with biological networks using subcellular localization annotation. Bioinformatics. 2007;23(8):1040-2.

16. Blanco-Melo D, Nilsson-Payant BE, Liu WC, Uhl S, Hoagland D, Moller R, Jordan TX, Oishi K, Panis M, Sachs D, et al. Imbalanced Host Response to SARS-CoV-2 Drives Development of COVID-19. Cell. 2020;181(5):1036-45 e1039.

17. de Hoon MJ, Imoto S, Nolan J, Miyano S. Open source clustering software. Bioinformatics. 2004;20(9):1453-4.

18. Luo W, Pant G, Bhavnasi YK, Blanchard SG Jr, Brouwer C. Pathview Web: user friendly pathway visualization and data integration. Nucleic Acids Res. 2017;45(W1):W501-8.

19. Du ZP, Wu BL, Wang SH, Shen JH, Lin XH, Zheng CP, Wu ZY, Qiu XY, Zhan XF, Xu LY, et al. Shortest path analyses in the protein-protein interaction network of NGAL (neutrophil gelatinase-associated lipocalin) overexpression in esophageal squamous cell carcinoma. Asian Pac J Cancer Prev. 2014;15(16):6899-904.

20. Santolini M, Barabasi AL. Predicting perturbation patterns from the topology of biological networks. Proc Natl Acad Sci U S A. 2018;115(27):E6375-83.

21. Hung MC, Link W. Protein localization in disease and therapy. J Cell Sci. 2011;124(Pt 20):3381-92.

22. Wang X, Li S. Protein mislocalization: mechanisms, functions and clinical applications in cancer. Biochim Biophys Acta. 2014;1846(1):13-25.

23. Gordon DE, Jang GM, Bouhaddou M, Xu J, Obernier K, White KM, O'Meara MJ, Rezelj VV, Guo JZ, Swaney DL, et al. A SARS-CoV-2 protein interaction map reveals targets for drug repurposing. Nature. 2020;583(7816):459-68.

24. Chen B, Fan W, Liu J, Wu FX. Identifying protein complexes and functional modules-from static PPI networks to dynamic PPI networks. Brief Bioinform. 2014;15(2):177-94.

25. Wang J, Peng X, Peng W, Wu FX. Dynamic protein interaction network construction and applications. Proteomics. 2014;14(4-5):338-52.

26. Cheng F, Desai RJ, Handy DE, Wang R, Schneeweiss S, Barabasi AL, Loscalzo J. Network-based approach to prediction and population-based validation of in silico drug repurposing. Nat Commun. 2018;9(1):2691.

27. Anighoro A, Bajorath J, Rastelli G. Polypharmacology: challenges and opportunities in drug discovery. J Med Chem. 2014;57(19):7874-87. 
28. Lu DY, Wu HY, Yarla NS, Xu B, Ding J, Lu TR. HAART in HIV/AIDS Treatments: Future Trends. Infect Disord Drug Targets. 2018;18(1):15-22.

29. Marrocco I, Romaniello D, Yarden Y. Cancer Immunotherapy: The Dawn of Antibody Cocktails. Methods Mol Biol. 2019;1904:11-51.

30. Lang T, Liu Y, Zheng Z, Ran W, Zhai Y, Yin Q, Zhang P, Li Y. Cocktail Strategy Based on SpatioTemporally Controlled Nano Device Improves Therapy of Breast Cancer. Adv Mater. 2019;31(5):e1806202.

31. Pollyea DA. New drugs for acute myeloid leukemia inspired by genomics and when to use them. Hematology Am Soc Hematol Educ Program. 2018;2018(1):45-50.

32. Zimmer A, Tendler A, Katzir I, Mayo A, Alon U. Prediction of drug cocktail effects when the number of measurements is limited. PLoS Biol. 2017;15(10):e2002518.

33. Lee HW, Sa JK, Gualberto A, Scholz C, Sung HH, Jeong BC, Choi HY, Kwon GY, Park SH. A phase II trial of tipifarnib for patients with previously-treated, metastatic urothelial carcinoma harboring HRAS mutations. Clin Cancer Res 2020.

34. Mine N, Yamamoto S, Saito N, Yamazaki S, Suda C, Ishigaki M, Kufe DW, Von Hoff DD, Kawabe T. CBP501-calmodulin binding contributes to sensitizing tumor cells to cisplatin and bleomycin. Mol Cancer Ther. 2011;10(10):1929-38.

35. Chen BJ, Wu YL, Tanaka Y, Zhang W. Small molecules targeting c-Myc oncogene: promising anticancer therapeutics. Int J Biol Sci. 2014;10(10):1084-96.

\section{Table}

Table 1. ACE2 interacting proteins from database 


\begin{tabular}{|c|c|c|c|c|}
\hline Gene ID & $\begin{array}{l}\text { Official } \\
\text { Symbol }\end{array}$ & Full name & $\begin{array}{l}\text { Experimental } \\
\text { System }\end{array}$ & Pubmed ID \\
\hline 801 & CALM1 & $\begin{array}{l}\text { calmodulin } 1 \text { (phosphorylase } \\
\text { kinase, delta) }\end{array}$ & $\begin{array}{l}\text { Affinity } \\
\text { Capture- } \\
\text { Western }\end{array}$ & 18070603 \\
\hline 183 & AGT & $\begin{array}{l}\text { angiotensinogen (serpin } \\
\text { peptidase inhibitor, clade A, } \\
\text { member 8) }\end{array}$ & $\begin{array}{l}\text { Biochemical } \\
\text { Activity }\end{array}$ & 10969042 \\
\hline 14 & AAMP & $\begin{array}{l}\text { angio-associated, migratory cell } \\
\text { protein }\end{array}$ & $\begin{array}{l}\text { Affinity } \\
\text { Capture-MS }\end{array}$ & 26186194 \\
\hline 847 & CAT & catalase & $\begin{array}{l}\text { Co- } \\
\text { fractionation }\end{array}$ & 26344197 \\
\hline 51477 & ISYNA1 & inositol-3-phosphate synthase 1 & $\begin{array}{l}\text { Co- } \\
\text { fractionation }\end{array}$ & 26344197 \\
\hline 8847 & DLEU2 & $\begin{array}{l}\text { deleted in lymphocytic leukemia } 2 \\
\text { (non-protein coding) }\end{array}$ & $\begin{array}{l}\text { Affinity } \\
\text { Capture-RNA }\end{array}$ & 28977802 \\
\hline 3265 & HRAS & $\begin{array}{l}\text { Harvey rat sarcoma viral } \\
\text { oncogene homolog }\end{array}$ & $\begin{array}{l}\text { Proximity } \\
\text { Label-MS }\end{array}$ & 30639242 \\
\hline 43740568 & S & Spike glycoprotein & $\begin{array}{l}\text { Co-crystal } \\
\text { Structure }\end{array}$ & 32132184 \\
\hline 340024 & SLC6A19 & $\begin{array}{l}\text { solute carrier family } 6 \text { (neutral } \\
\text { amino acid transporter), member } \\
19\end{array}$ & $\begin{array}{l}\text { Co-crystal } \\
\text { Structure }\end{array}$ & 32132184 \\
\hline 7431 & VIM & vimentin & $\begin{array}{l}\text { Affinity } \\
\text { Capture- } \\
\text { Western }\end{array}$ & 26801988 \\
\hline \multirow[t]{2}{*}{1670} & \multirow[t]{2}{*}{ DEFA5 } & \multirow{2}{*}{$\begin{array}{l}\text { defensin, alpha 5, Paneth cell- } \\
\text { specific }\end{array}$} & \multirow{2}{*}{$\begin{array}{l}\text { Reconstituted } \\
\text { Complex }\end{array}$} & DOI:10.1101 \\
\hline & & & & $/ 2020.03 .29 .013490$ \\
\hline 51738 & GHRL & ghrelin/obestatin prepropeptide & & 11815627 \\
\hline
\end{tabular}

\section{Figures}




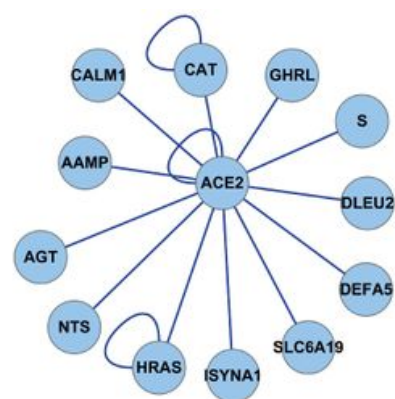

C

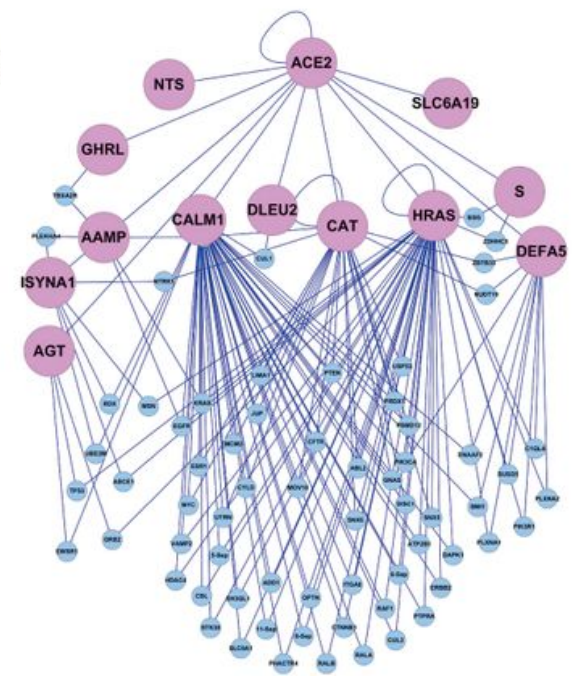

B

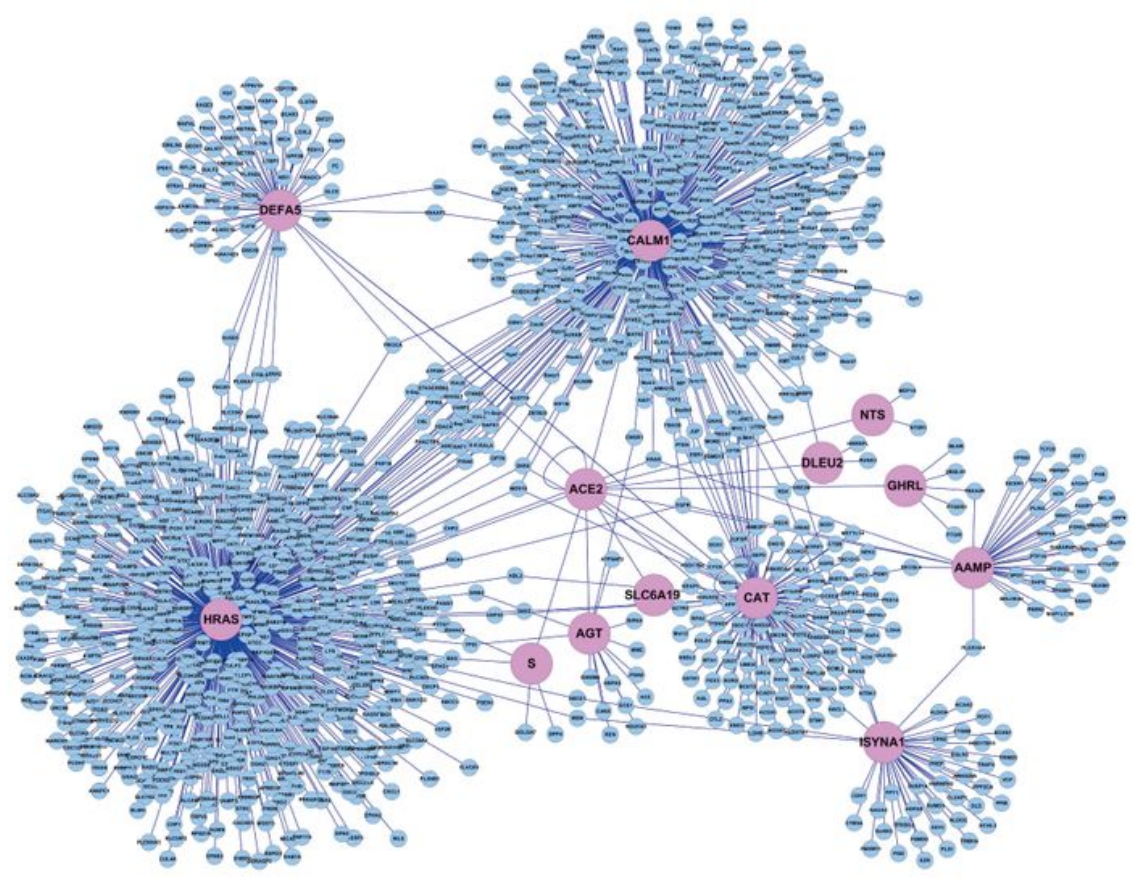

Figure 1

PPINs for ACE2 and its interacting proteins. A. The reported ACE2 interacting proteins. B. The "ACE2 FullPPIN" is consist of ACE2 and its interacting proteins with all their public reported interacting proteins. C. The "ACE2 Core-PPIN" was originated from the Full-PPIN by moving the nodes with only one link, to illustrate ACE2 and its interacting proteins are connected with many partner proteins.

A

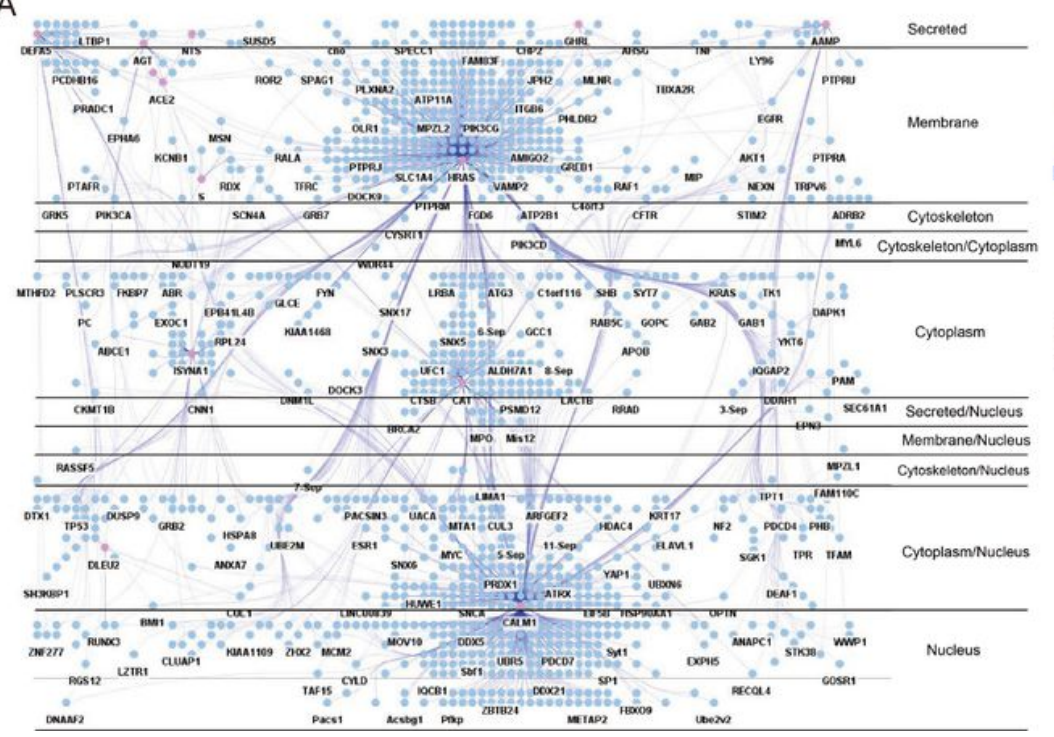

B

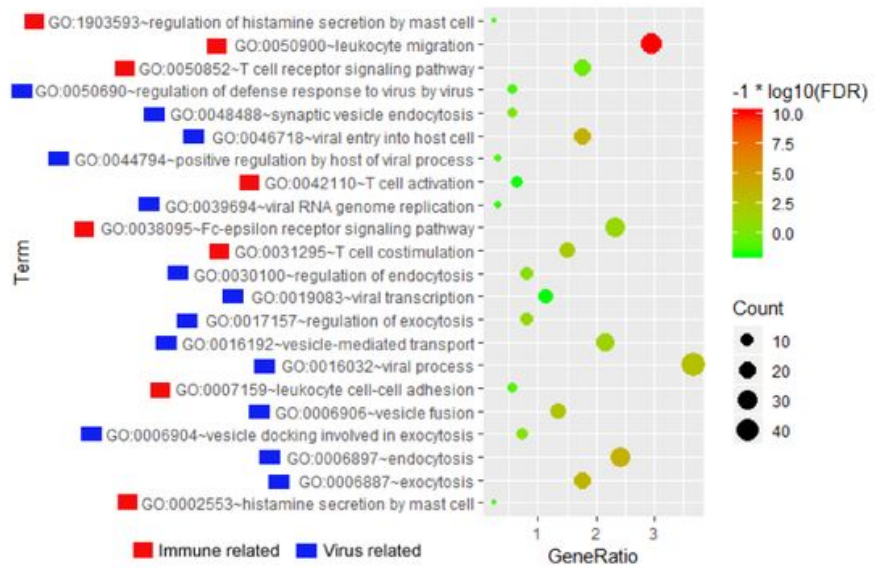

Figure 2 
A. Subcellular layers of "ACE2 Full-PPIN", which is separated into eleven layers while maintaining their protein interactions. ACE22 and its interacting proteins are shown in pink color. B. Gene Ontology "Biological Process" enrichment of the Full-PPIN shows significant virus activity and immune related terms.

A

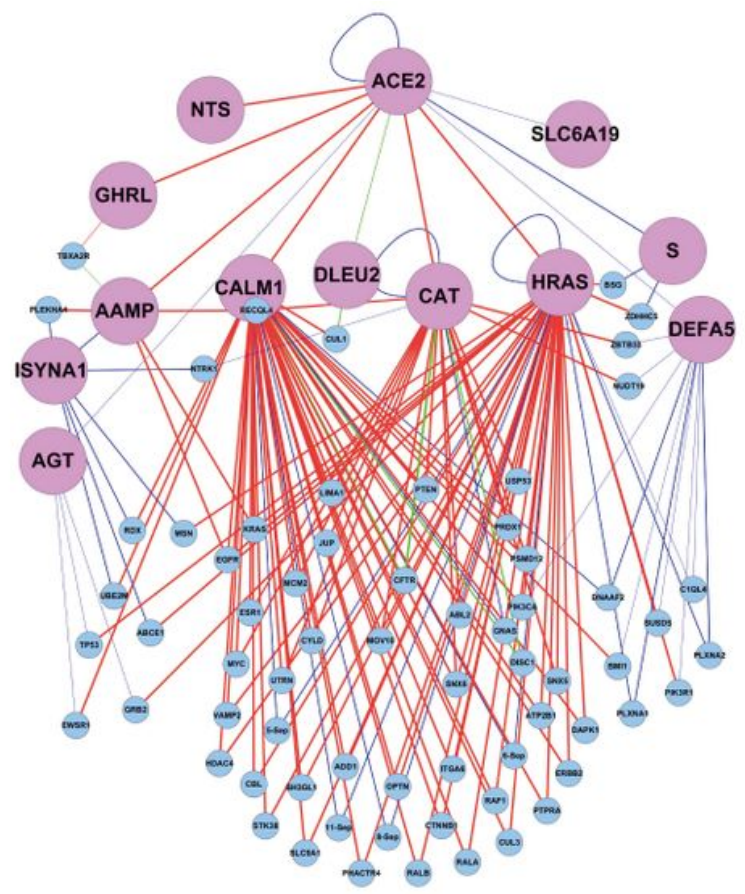

C

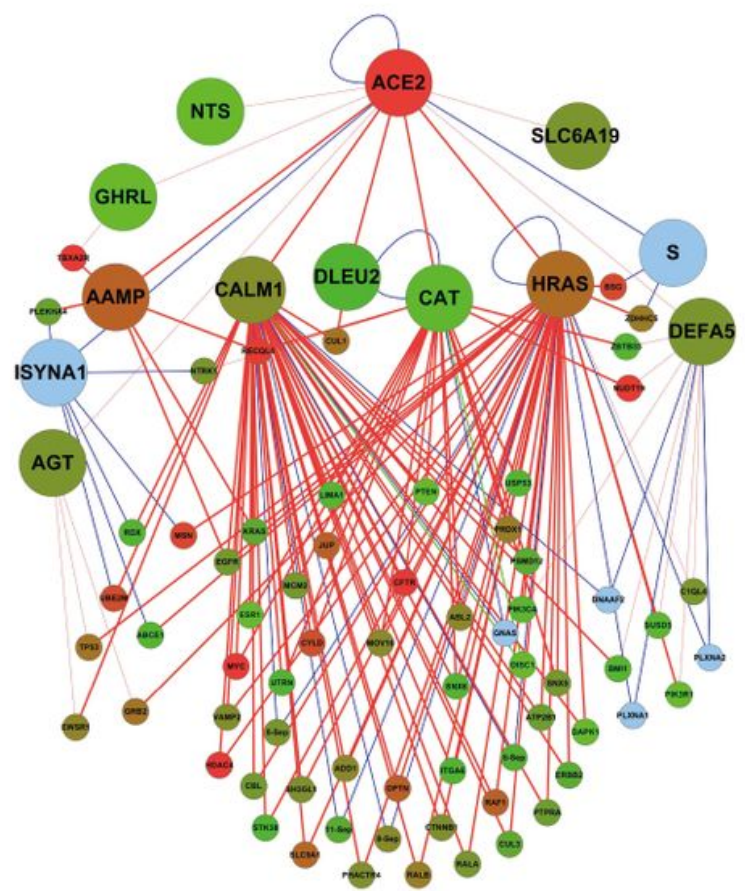

B
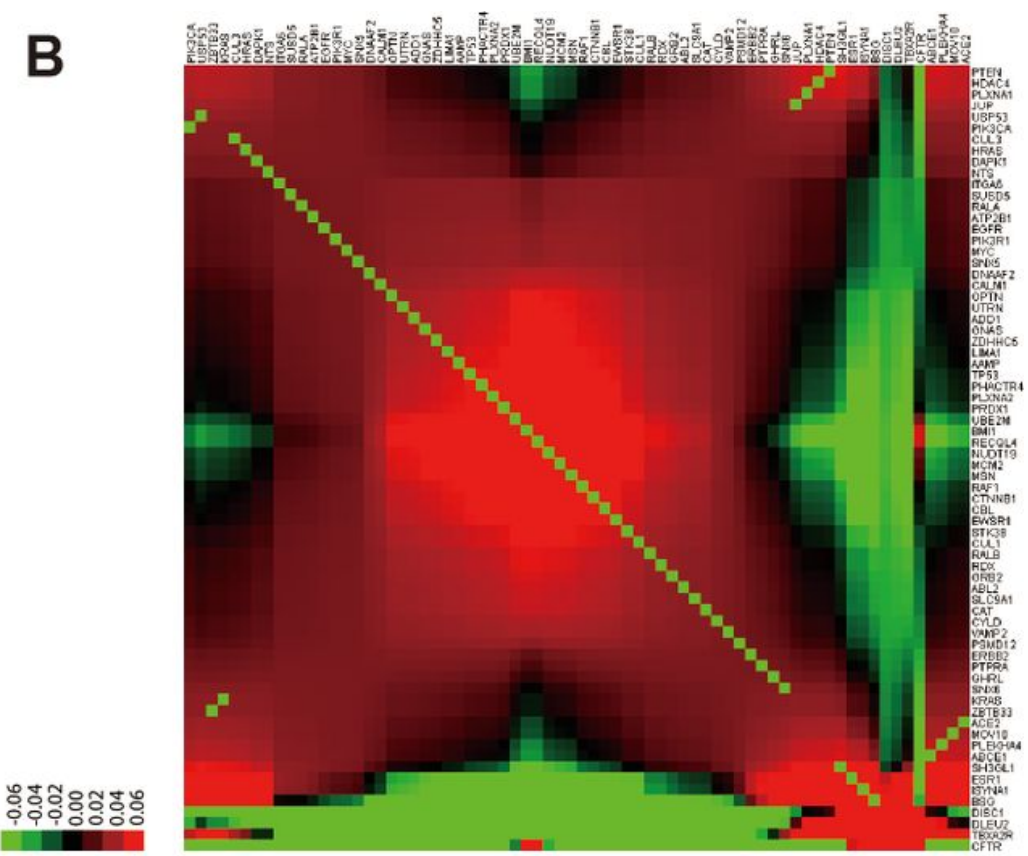

D

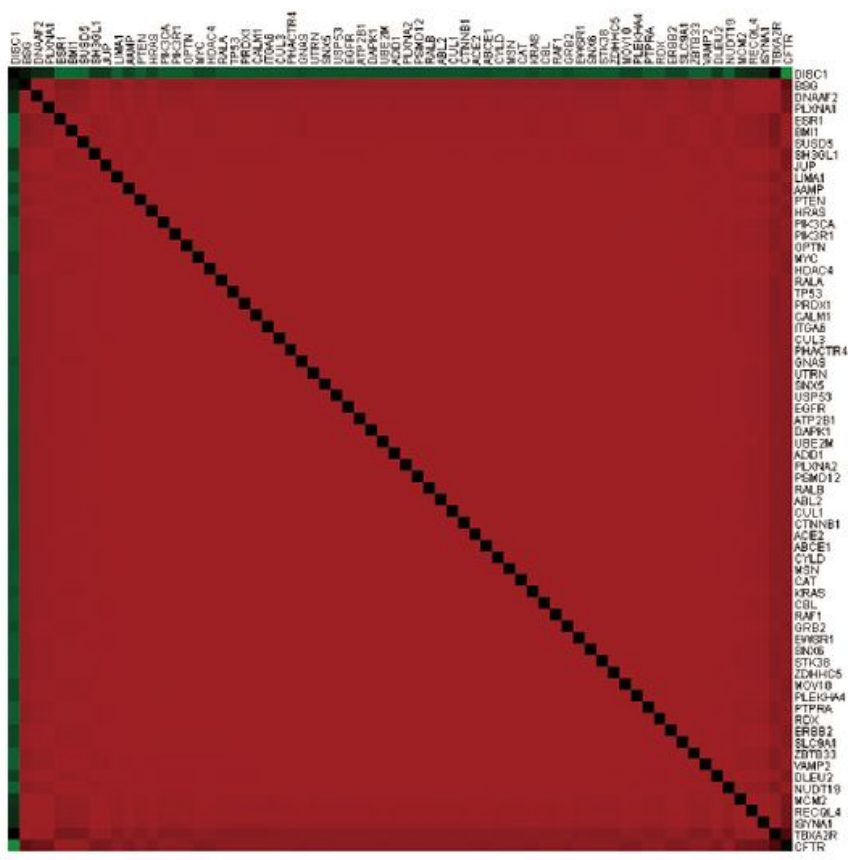

\section{Figure 3}

Expression fold-change and co-expression correlation of proteins in the "ACE2 Core-PPIN" for normal lung and that treated with SARS-CoV-2. A. The co-expression relationship for "ACE2 Core-PPIN" in normal lung cells. B. The heatmap of co-expression relationship for proteins in "ACE2 Core-PPIN" in normal lung cells. 
C. The expression fold-change for the Core-PPIN in lung cells treated with SARS-CoV-2. D. The heatmap of co-expression relationship for "ACE2 Core-PPIN" in lung cells treated with SARS-CoV-2.

A

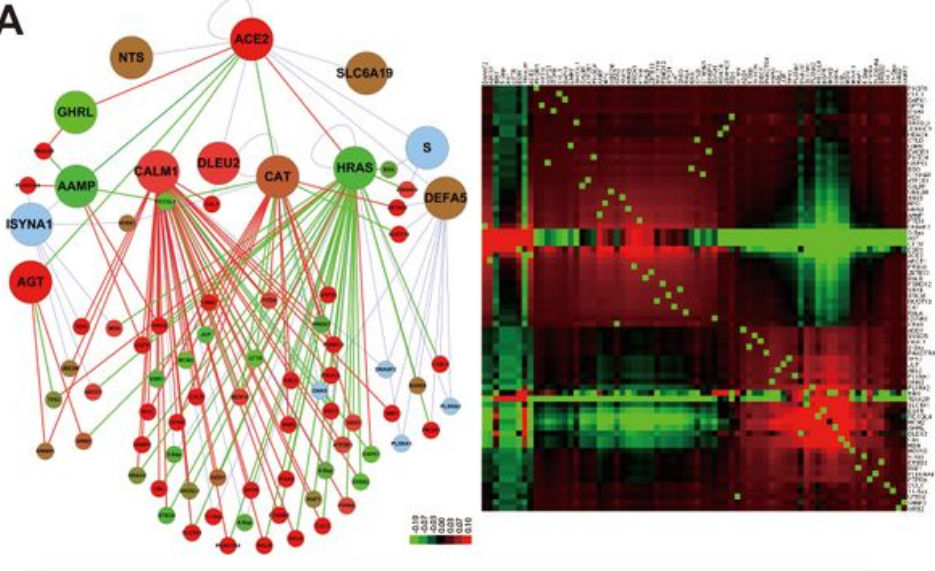

Mock

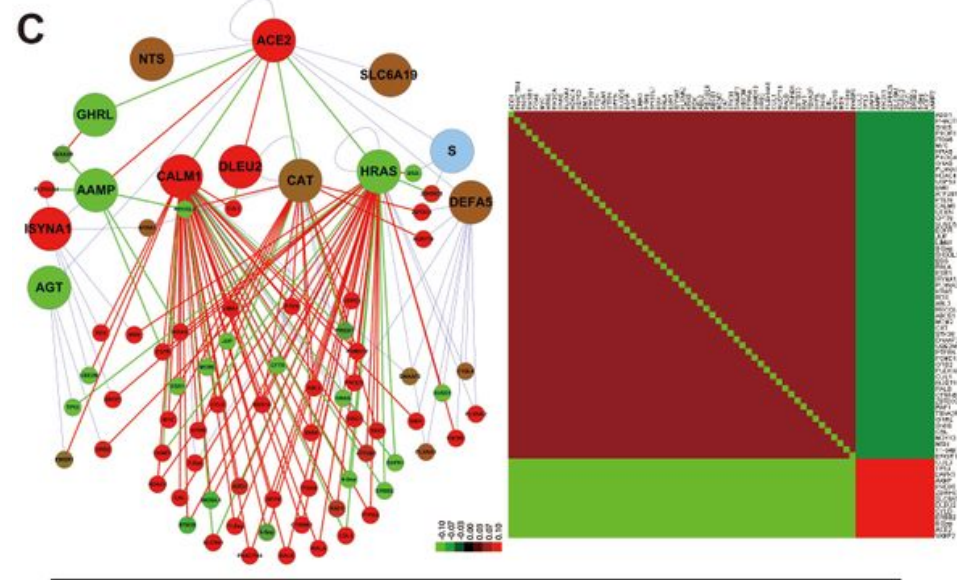

IFNB_6h

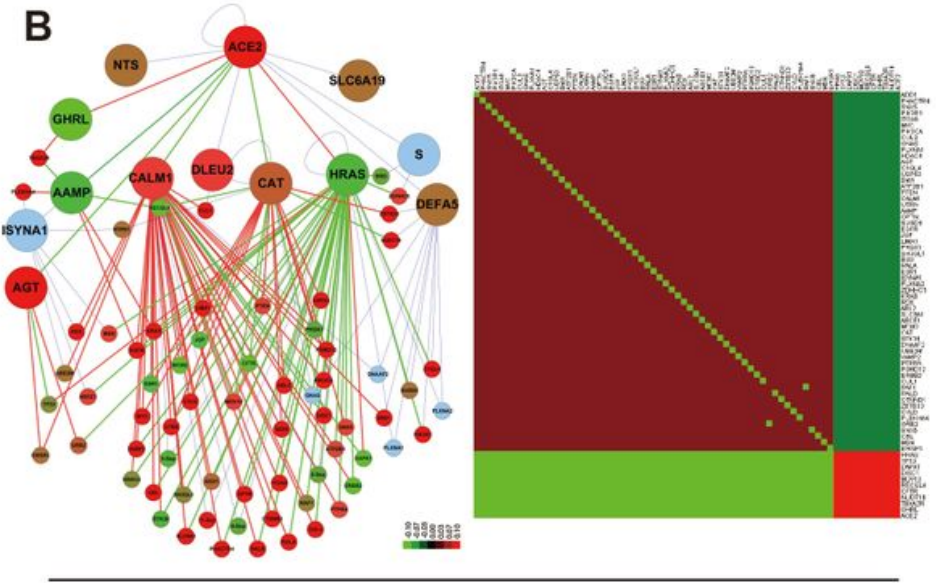

IFNB_4h

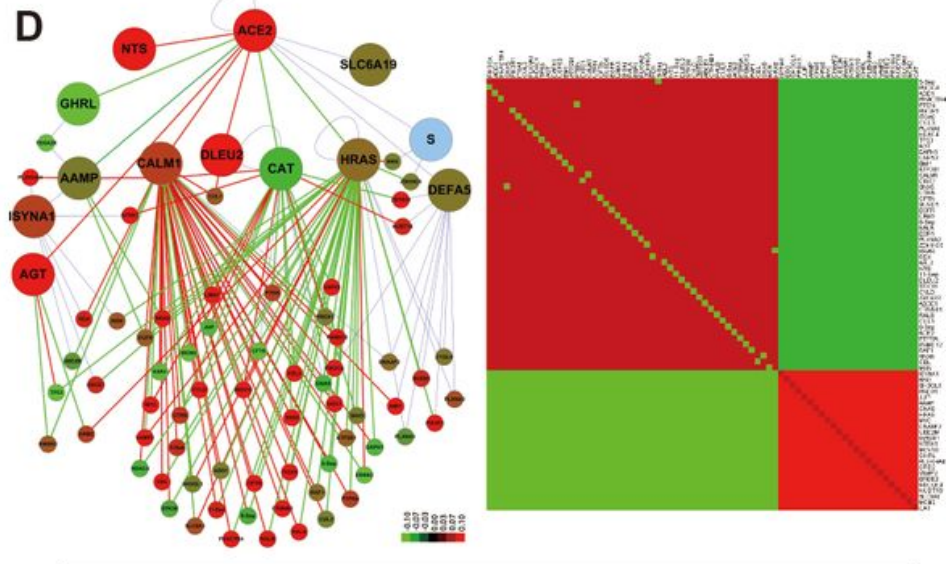

IFNB_12h

\section{Figure 4}

Fold-change in expression and Pearson correlation matrix of proteins in the "ACE2 Core-PPIN" for normal lung cells treated with INFN at different time points. (A-D, left panel)The expression fold-change for "ACE2 Core-PPIN" in normal lung cells with mock treatment or with INFN at $4 \mathrm{~h}, 6 \mathrm{~h}$ and $12 \mathrm{~h}$, with the mock as the control. (A-D, right panel)The heatmap of co-expression relationship for "ACE2 Core-PPIN" in normal lung cells. 

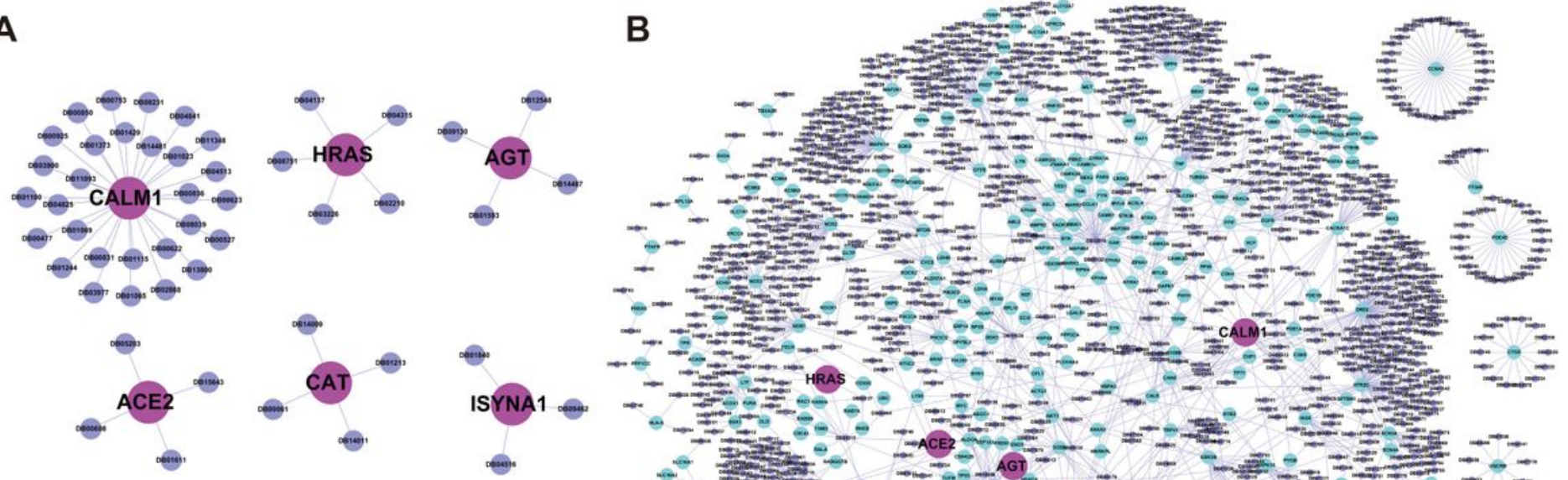

\begin{tabular}{|l|l|l|l|}
\hline $\begin{array}{l}\text { Protein Node } \\
\text { ESR1 }\end{array}$ & $\begin{array}{c}\text { Degree } \\
118\end{array}$ & $\begin{array}{l}\text { Drug Node } \\
\text { DB12010 }\end{array}$ & $\begin{array}{c}\text { Degree } \\
\text { DRD2 }\end{array}$ \\
\hline AR & 109 & DB11638 & 24 \\
\hline AR & 81 & DB09130 & 16 \\
\hline ADRB2 & 70 & DB14487 & 14 \\
\hline HTR2C & 66 & DB01593 & 14 \\
\hline OPRM1 & 64 & DB14548 & 13 \\
\hline ESR2 & 58 & DB14533 & 13 \\
\hline MAPK14 & 56 & DB04315 & 13 \\
\hline DPP4 & 54 & DB12695 & 10 \\
\hline HSP90AA1 & 49 & DB01254 & 10 \\
\hline
\end{tabular}

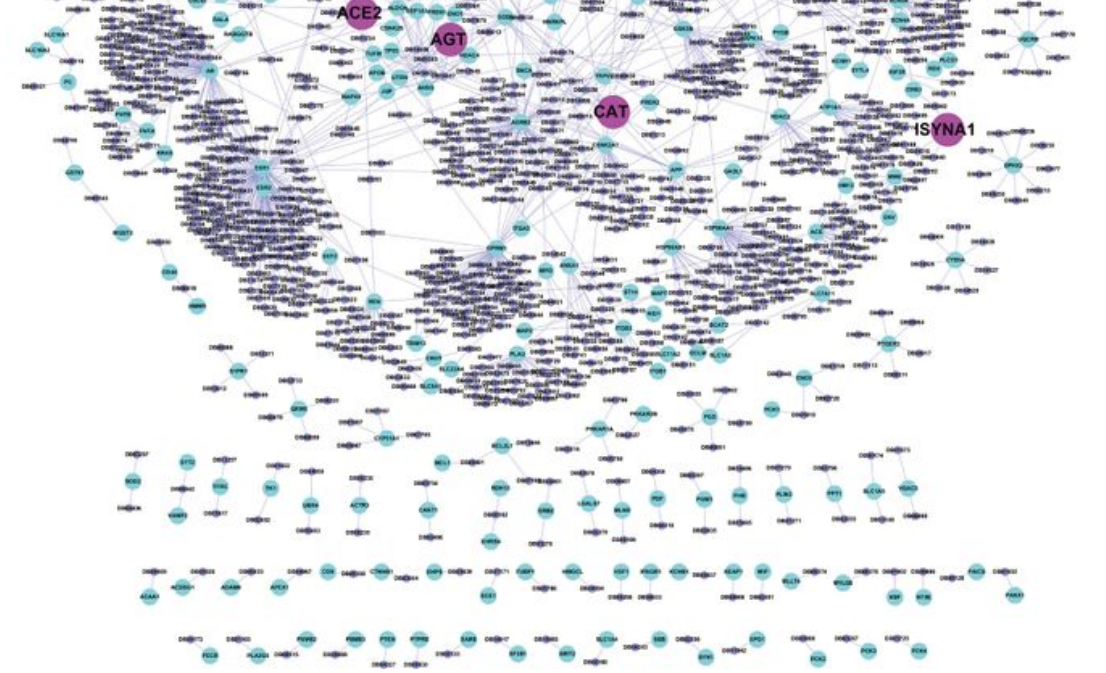

\section{Figure 5}

Drug-protein network for ACE2 and its interacting proteins. A. Reported drugs for ACE2 and its interacting proteins, respectively. B. Drugs target the proteins in "ACE2 Full-PPIN". ACE2 and its interacting proteins are indicated in light pink, their secondary interacting proteins are shown in light blue. Drugs are illustrated in light purple. C. The top 10 proteins and drugs have the highest number of edges.

\section{A}

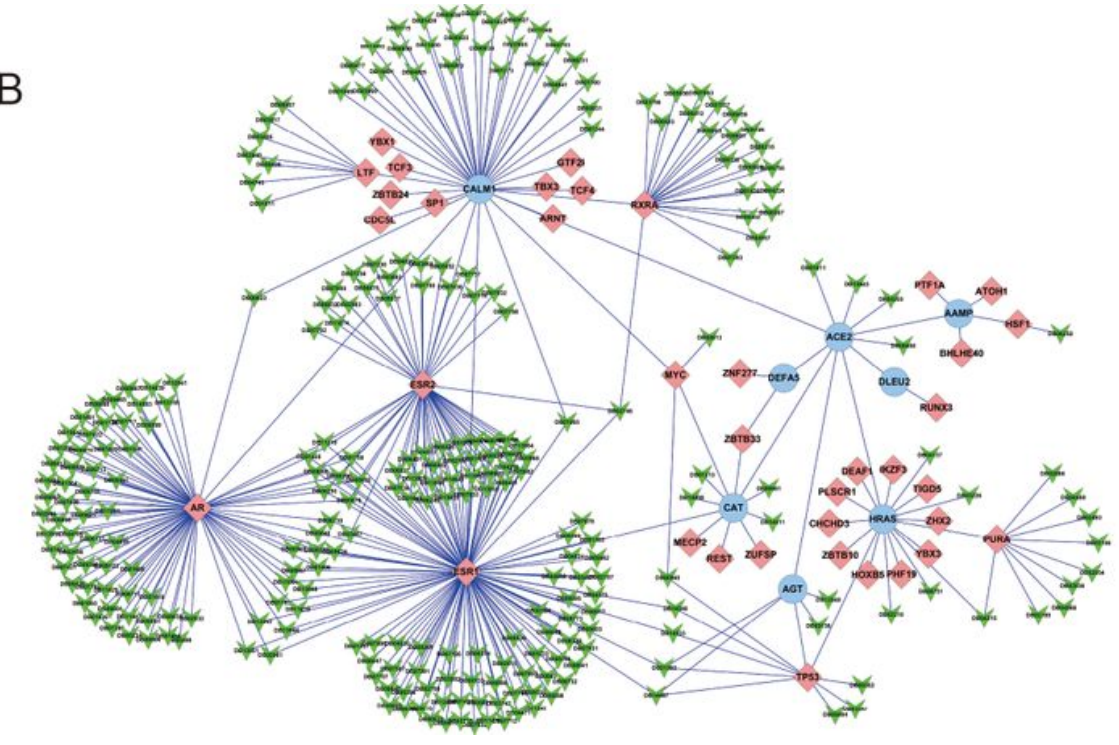

Figure 6 
The shortest paths for ACE2 and transcription factors and their drugs. A. All the possible shortest paths from ACE2 to transcription factors. The transcription factors are indicated in light pink. B. The drug network targets the shortest paths from ACE2 to transcription factors, suggesting the possible disturbing their signal transduction.

A

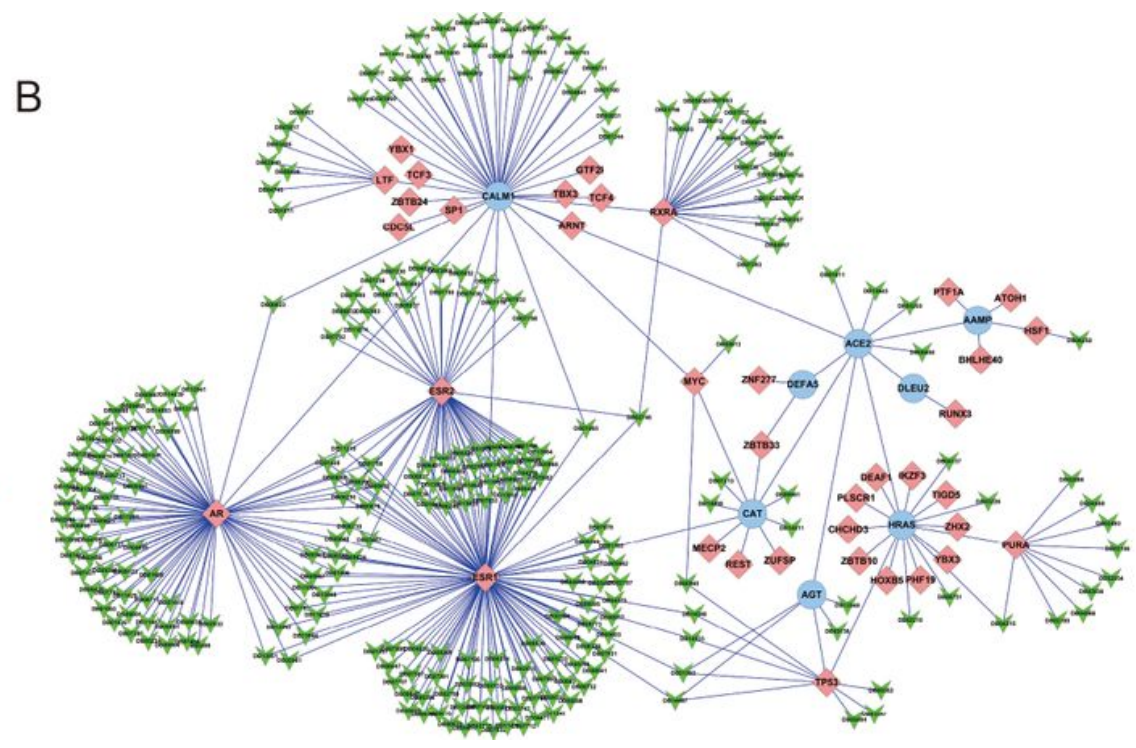

Figure 7

A propose model for drugs disrupt the biological activity caused by SARC-CoV-2 mediated by ACE2 and its cascaded interacting proteins.

\section{Supplementary Files}

This is a list of supplementary files associated with this preprint. Click to download.

- Additionalfile1.pdf

- Additionalfile2.xlsx

- Additionalfile3.pdf

- Additionalfile4.pdf

- Additionalfile5.csv

- Additionalfile6.pdf 